\title{
INTERVALLEY TRANSFER OF ELECTRONS IN ZnS-TYPE THIN FILM ELECTROLUMINESCENT DEVICES
}

\author{
H. ZHAO, Y. WANG* ${ }^{*}$ Z. XU AND X. XU \\ Institute of Optoelectronic Technology, Northern Jiaotong University \\ Beijing 100044, P.R.China
}

(Received February 16, 1999; revised version May 11, 1999)

\begin{abstract}
Based on the calculation about intervalley scattering rates in $\mathrm{ZnS}$, the intervalley transfer process in ZnS-type thin film electroluminescent devices is investigated through the Monte Carlo simulation. The transient time of intervalley transfer is about $0.2-0.3 \mathrm{ps}$, it coincides with that of electron average energy. Intervalley distribution shifts to high valleys as the electric field increased. The electron kinetic energy distributions in different valleys are also gained. We propose that high valleys could store energies, which could prolong the decay of the electron average energy as the field was removed. These results conld be used as the basic data on the study of electroluminescent process and the citation of valley parameters in analytic models should be carefully considered.
\end{abstract}

PACS numbers: 72.20.-i, 78.60.-b

\section{Introduction}

Thin film electroluminescent devices (TFELDs) have become of great interest since they offer a possible means of achieving a high-resolution, light-weight, compact video display panel for computer terminals or television screens [1]. The first commercial TFELD was introduced by sharp in 1983. Nowadays, monochrome TFELDs have been used in many fields. The basic mechanism of electroluminescence (EL) is based on high-field acceleration of electrons to optical energies at which luminescent centers, intentionally introduced into the host material, can be impact excited.

The electron transport process is one of the most important processes in EL since it determined how energetic the electrons were. This process is the result of two contradict trends of electrons: acceleration by electric field and scattering by several kinds of scattering processes. The latter influences the acceleration effect

\footnotetext{
*e-mail: yshwang@center.njtu.edu.cn
} 
seriously. ZnS has a multivalley band structure and the intervalley scattering is one of the main scattering mechanisms [2]. At the same time, intervalley scattering is accompanied by the conversion of electron's kinetic and potential energies. This will influence the kinetic energy distribution of electrons and consequently the EL process. As no reports on the electron intervalley transfer process is found in literature, we investigated this process through the Monte Carlo simulation.

\section{Theoretical analysis}

There are three energy valleys in the conduction band of $\mathrm{ZnS}: \Gamma, L$, and $X$ valley. They could be described as a nonparabolic valley model

$$
E\left(1+\alpha_{n} E\right)=\frac{\hbar^{2} k^{2}}{2 m_{n}^{*}} \quad\left(n=\Gamma^{\prime}, L, X\right)
$$

in which $\alpha_{n}$ and $m_{n}^{*}$ are the nomparabolic factor and effective mass in $n$-valley, respectively. The bottom energies of these valleys are different. We set the bottiom of $\Gamma$ valley as the zero point of potential energy, then the electrons in $L$ and $X$ valleys have potential energies as well as kinetic energies. Electron intervalley scattering will be accompanied by the conversion of kinetic and potential energies.

Intervalley scattering is limited by energy and momentum conservation, so the participation of phonons is necessary. In the nomparabolic valley model, intervalley scattering rate is [3]

$$
S(E)=\frac{D_{\mathrm{if}}^{2} Z_{\mathrm{f}}\left(m_{\mathrm{i}}^{*}\right)^{3 / 2}}{\sqrt{2} \pi \hbar^{2} \rho E_{\text {phonon }}}\left(1+2 \alpha_{\mathrm{i}} \Delta E\right) \sqrt{\Delta E\left(1+\alpha_{\mathrm{i}} \Delta E\right)}\left(\begin{array}{c}
N_{q} \\
N_{q+1}
\end{array}\right) .
$$

$E, D_{\mathrm{if}}, Z_{\mathrm{i}}, \rho, \alpha_{\mathrm{i}}, m_{\mathrm{i}}^{*}$ stand for the electron energy, intervalley deformation potential between initial and final valleys, number of equivalent final valleys, longitudinal acoustic velocity, momparabolic factor of initial valley, and effective mass of electrons in initial valley, respectively. $\Delta E=E \pm E_{\text {phonon }}-E_{\text {if }}$, in which $E_{\text {phonon }}$ is the energy of phonon and $E_{\text {if }}$ - the energy difference between the bottoms of initial and final valleys. $N_{q}$ and $N_{q+1}$ are phonon numbers which are assumed to follow the Bose-Einstein distribution. There are two different processes in each kind of intervalley scattering, absorption and emission of phonon. $N_{q}$ and "+" correspond to absorption and, $N_{q+1}$, "-."- the emission of phonon.

The rates of intervalley scattering in $\mathrm{ZnS}$ are calculated in different electron energies under $300 \mathrm{~K}$. The parameters used here could be found in Ref. [4]. The results are shown in Fig. 1. Each curve is the total rate of the absorption and emission phonon processes. The raties increase with electron energy. Since $L$ valley has the biggest number of equivalent valleys, 4 , the rates toward to $L$ valley is higher than to others. The bottom of $\Gamma$ valley is the lowest, so, because of the potential energy difference, the scatiering rates from $\Gamma$ valley to $L$ and $X$ valley must pass a threshold energy of about $1.4 \mathrm{eV}$.

The transport process in solid could be described by the Boltzmann equation. However, this equation is difficult to be solved, especially in high-field cases, as in TFELD. For the investigation of EL process, there are mainly two methods, lucky drift model $[5,6]$ and the Monte Carlo simulation [7, 8]. The former seeks for the relations among macroscopic quantities phenomenally and the latter simulates 


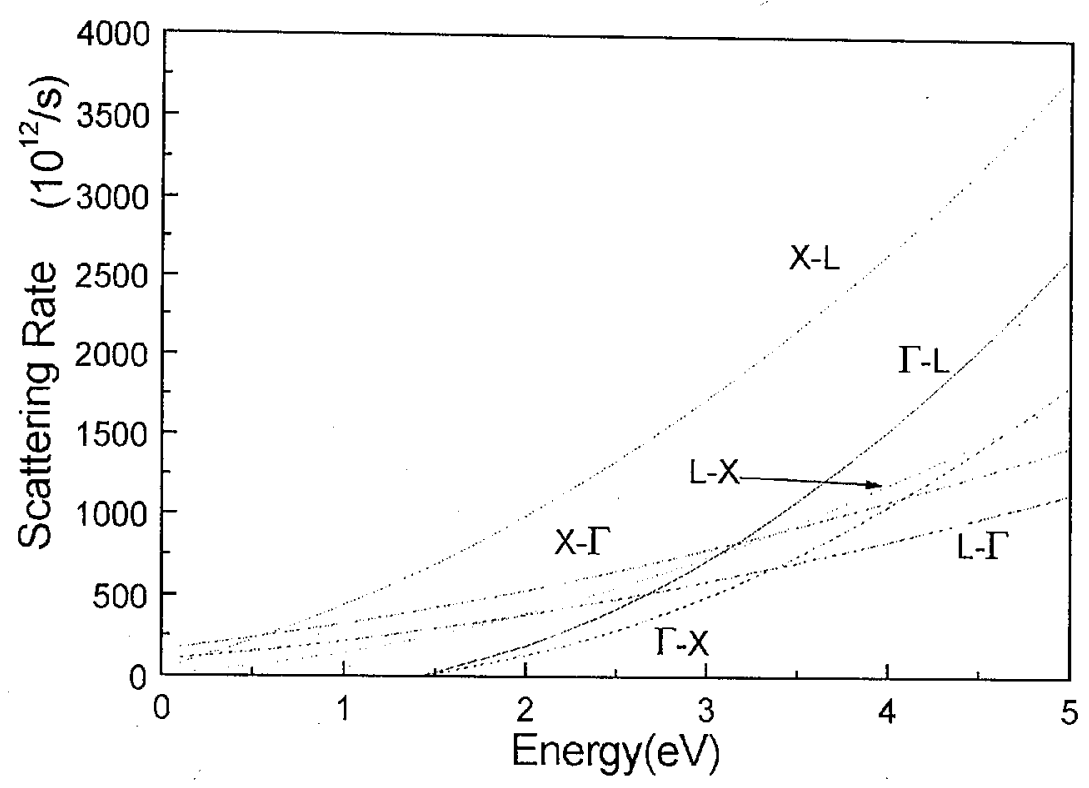

Fig. 1. Intervalley scattering rates of electrons in $\mathrm{ZnS}$.

the microscopic process of electron motion in materials. We have investigated the transient acceleration process in TFELD using the Monte Carlo method [9]. To investigate the intervalley transfer process, which composes a series of microscopic processes, the Monte Carlo simulation is the most effective tool.

The Monte Carlo simulation is a simulation of the motion of individual carriers as they move under the influence of external fields and scattered by various mechanisms. This motion is composed by a serial of "free flight" processes terminated by the scattering events. During the "free flight" process, the influence of scattering mechanisms is not considered and the motion could be described as

$$
\frac{\mathrm{d}}{\mathrm{d} t}(\hbar k)=-e E,
$$

where $\hbar$ is the reduced Planck constant, $e$ is the electronic charge, $\boldsymbol{E}$ is the electric field strength and $k$ is the wave vector. The free flight time is chosen stochastically according to the total scattering rate

$$
t_{\mathrm{r}}=-\frac{1}{\Gamma} \ln \left(r_{1}\right)
$$

where $\Gamma$ is the total scattering rate (the sum of rates of all scattering mechanisms considered) and $r_{1}$ is a random number generated by the program and is uniformly distributed between 0 and 1 .

At the end of this free flight $\left(t=t_{\mathrm{r}}\right)$, another random number uniformly distributed between 0 and $1, r_{2}$, is generated by the program to choose a certain kind of scattering from all of the scattering mechanisms. If there are $n$ kinds of scattering mechanisms and the rate of $i$-th is $W_{i}$, then the probability of the 
occurrence of $m$-th scattering is

$$
W_{m} / \sum_{i=1}^{n} W_{i} .
$$

If

$$
\sum_{i=1}^{m-1} W_{i} / \sum_{i=1}^{n} W_{i}<r_{2} \leq \sum_{i=1}^{m} W_{i} / \sum_{i=1}^{n} W_{i},
$$

the $m$-th scattering mechanism is chosen.

Once a scattering mechanism has been chosen, the momentum and the energy of the electron are updated as determined by the specific mechanism that has been selected. Then the next "free flight" process begins until it is again terminated by the next scattering event. This cycle is repeated many times. The motion of an electron in phosphor layer is thus simulated.

In our Monte Carlo simulation, the scattering processes caused by acoustic phonons, polar optical phonons, and ionized impurities as well as intervalley scattering are considered. The intervalley scattering process has been discussed above. The rates of the other three kinds of the scattering processes and the relations between final and initial states have been investigated and reported elsewhere [10]. The number of simulated electrons is 50000 , which is large enough to ensure the accuracy and repeatability of the results.

\section{Results and discussions}

\subsection{Transient process of electron intervalley transfer}

When a voltage is applied to TFELD, the electrons captured in the insulator-phosphors interface enter the phosphors layer through tunnel emission [11]. Most of these initial electrons are in $\Gamma$ valley since energies are lower there. Under the electric field, these electrons become energetic and then begin to transfer to other valleys. The number of electrons in each valley varies with time until the dynamic balance is reached, as shown in Fig. 2. The transient time of intervalley transfer is about $0.2-0.3 \mathrm{ps}$. The transient increase in average kinetic energy is also shown in this figure and this transient time coincides with that of intervalley transfer. We have studied these transient processes in different electric fields $(0.5-5.0 \mathrm{eV})$ and gained similar results. Figure 2 shows the results in the case of $2.0 \mathrm{MV} / \mathrm{cm}$.

\subsection{The influence of electric field on intervalley distribution}

Although the transient processes of intervalley transfer in different fields are similar, the steady distributions vary with electric fields. Figure 3 shows the steady value of electron numbers in each valley and average kinetic energy of electrons with the variation of electric fields. The electron number in $\Gamma$ valley decreases with the field. At the same time, the numbers in $L$ and $X$ increase. This shows that the electrons transfer to higher valleys at higher electric field. It is worthy to mention that the variation is more rapidly at lower field. When the field reaches 


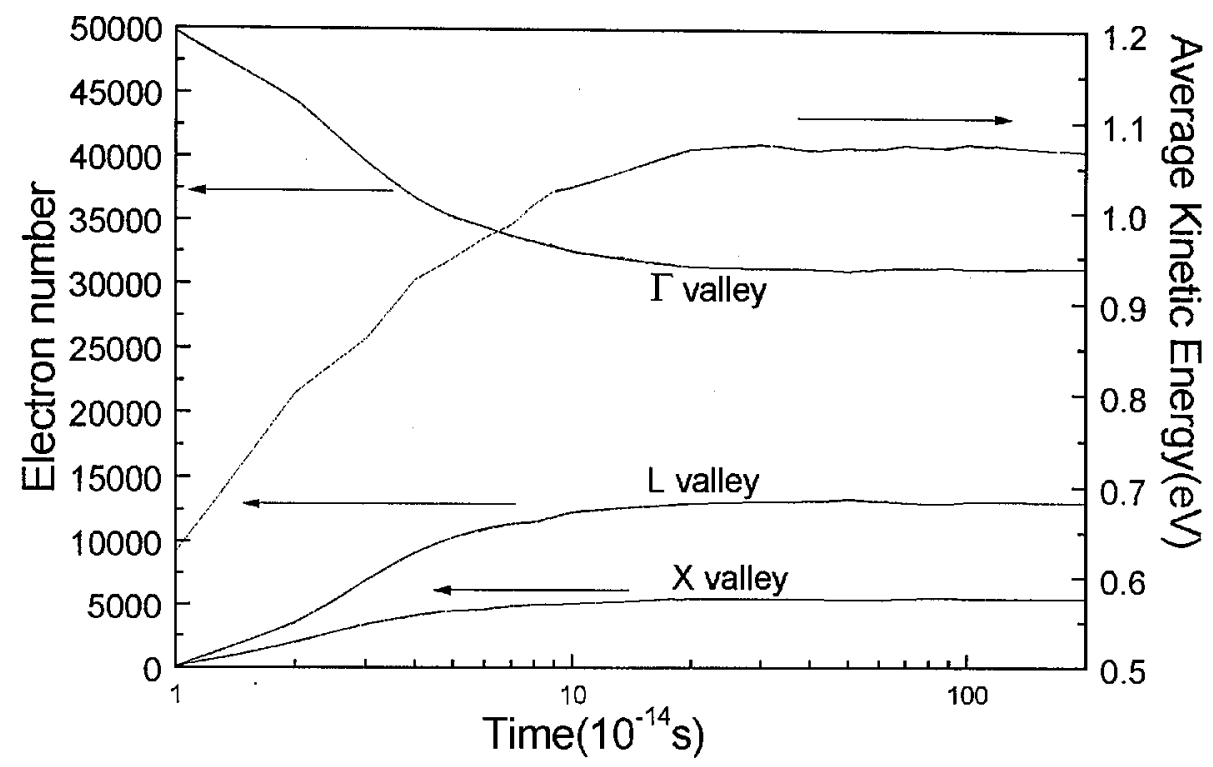

Fig. 2. Transient processes of electron intervalley distribution and the increase in average kinetic energy (under $2.0 \mathrm{MV} / \mathrm{cm}$ electric field).

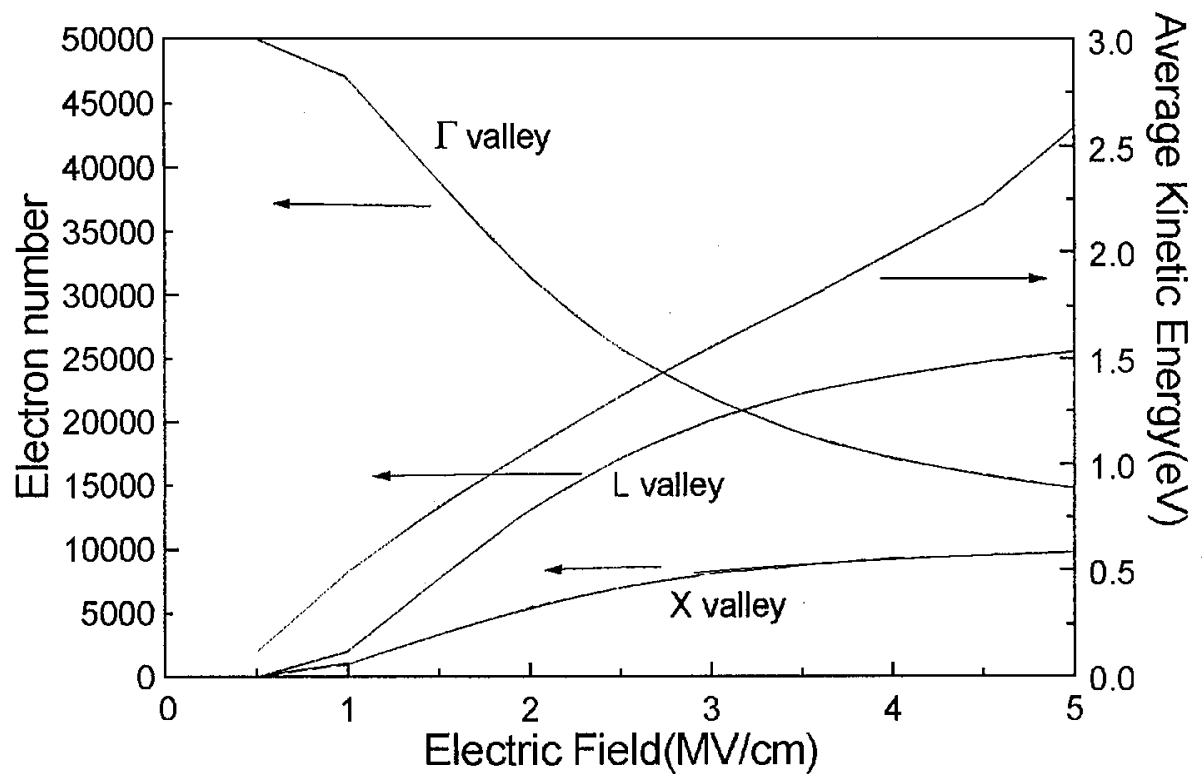

Fig. 3. Steady intervalley distributions and average kinetic energies in different electric fields.

4-5 MV/cm, the distribution tends to saturation. On the other hand, as shown in Fig. 3, the average kinetic energy increases steadily with the field and no saturation appears at $4-5 \mathrm{MV} / \mathrm{cm}$. 


\subsection{Electron kinetic energy distributions in different valleys}

Figure 4 shows the steady kinetic energy distributions in different valleys and the total distribution, e.g., the sum of the other distributions. All of these curves reach a peak around $0.3-0.4 \mathrm{eV}$ then decrease with energy. In the distribution curve of $\Gamma$ valley, another peak appears around $1.4 \mathrm{eV}$. The electron number changes slowly with energy before this peak and, after that, drops rapidly. This character does not appear in other valleys. As mentioned above, $1.4 \mathrm{eV}$ is the threshold

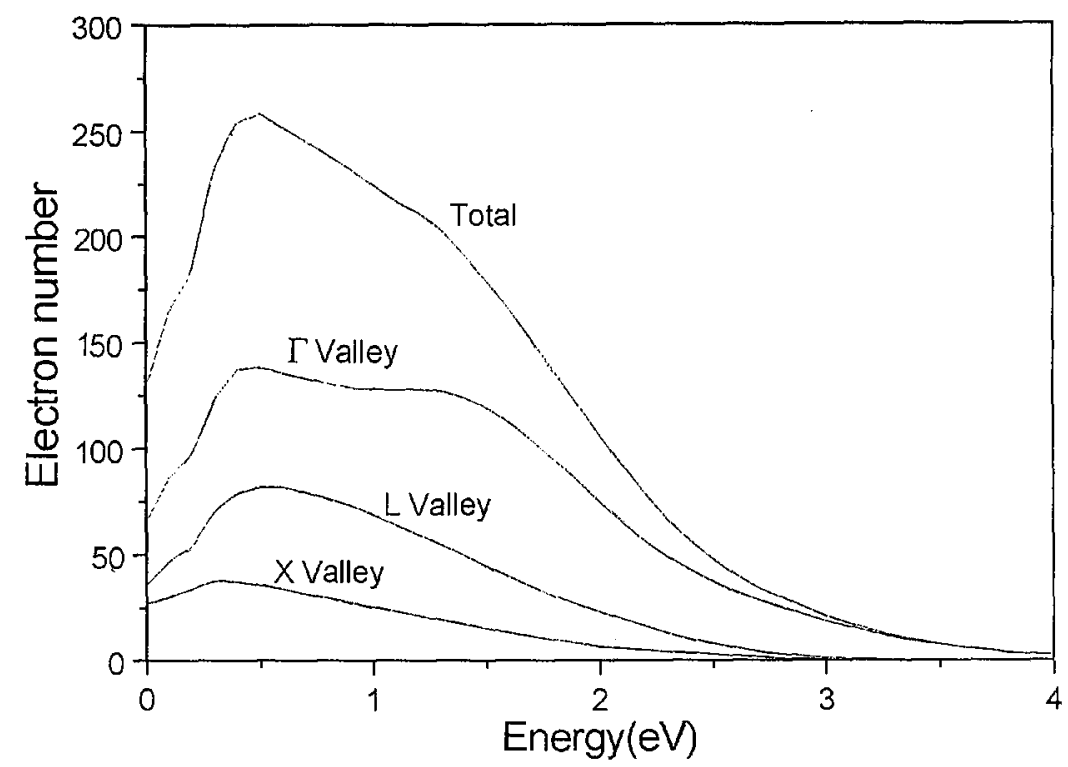

Fig. 4. Electron kinetic distributions in different valleys (under $2.0 \mathrm{MV} / \mathrm{cm}$ electric field).

energy of intervalley scattering from $\Gamma$ valley. So this character could be explained as follows. Before $1.4 \mathrm{eV}$, no intervalley scattering occurs, the electrons accumulate in $\Gamma$ valley. When the energy is higher than $1.4 \mathrm{eV}$, the electrons begin to transfer to other valleys due to intervalley scattering. The curve drops rapidly. Since the greatest part of electrons appears in $\Gamma$ valley (more than 60 percent at $2.0 \mathrm{MV} / \mathrm{cm}$ according to Fig. 3), the total distribution shows a similar behavior with that of $\Gamma$ valley.

\subsection{Energy storage effect of high valleys}

As mentioned above, intervalley scattering is accompanied by the conversion of electrons' kinetic and potential energies. A part of kinetic energy turns to potential and be stored in the upper valley as an electron transfers from low valley to high valley. When we remove the electric field, the dynamic balance is broken. Electrons transfer from high valleys to low valleys and the stored potential energies may be transformed into kinetic energies. Figure 5 shows this process. The system 


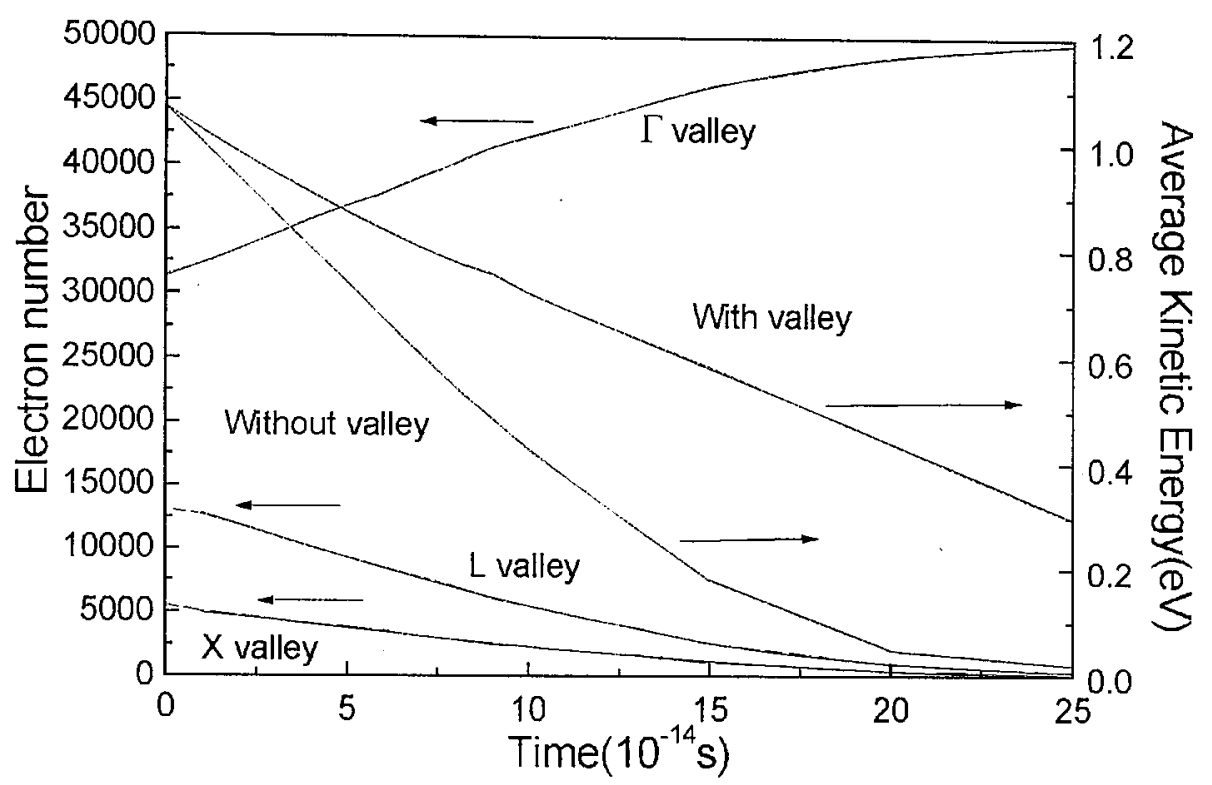

Fig. 5. Intervalley transfer process after the $2.0 \mathrm{MV} / \mathrm{cm}$ electric field is removed.

keeps balanced at $t=0$ under: $2.0 \mathrm{MV} / \mathrm{cm}$ field. Then the field is removed, and the system begins to move to a new balance. The number of electrons in $\Gamma$ valley increases and that of $L$ and $X$ valleys decreases. At about $0.25 \mathrm{ps}$, the system reaches the new balance. The decay of average kinetic energy is also shown ("with valley" curve in Fig. 5). For comparison, we calculated the decay of average kinetic energy without high valley distributions, the result is shown in Fig. 5 as "no valley" curve. The decay time of average kinetic energy is doubled by this storage effect. To the authors' knowledge, this is the first study on the energy storage effect of high valleys in 'TFELD. More investigations are still needed about the usage of this effect on the improvements of material, device structure, and operating conditions in TFELD.

\section{Conclusions}

Based on the analysis of intervalley scattering, the electron intervalley transfer process in ZnS-type TFELD is investigated quantitatively through the Monte Carlo simulation. The transient time of intervalley transfer process is about $0.2-0.3 \mathrm{ps}$, coincides with that of electron average kinetic energy. The steady electron intervalley distribution moves to high valley when the electric field is strengthened. This movement reaches a saturation point at $4-5 \mathrm{MV} / \mathrm{cm}$. The electron kinetic energy distributions in different valleys show different characters. This is caused by the properties of intervalley scattering. We propose that high valleys could store energies, this effect extends the decay process of average kinetic energy when the electric field is removed.

The results could be used as the basic data in the quantitative investigation of EL process. For example, when we studied the electron acceleration, impact 
excitation and electron multiplication processes through analytic method, parameters of $\Gamma$ valley are adopted $[12,13]$ since we believe that most of the electrons are in $\Gamma$ valley. In fact, based on our results, there are about 40 percent of electrons in $L$ and $X$ valleys under $2.0 \mathrm{MV} / \mathrm{cm}$ field. So the citation of $\Gamma$ valley parameters should be carefully considered. We propose that the weight of average parameters according to intervalley distribution should be introduced for $\Gamma$ valley parameters in such studies. The existing results about the EL process should be re-checked for the intervalley distribution, if necessary.

\section{Acknowledgments}

This work is supported by " 863 " Project of P.R. China and Paper Foundation of NJTU.

\section{References}

[1] Y.A. Ono, Electroluminescent Display, World Scientific, Singapore 1995, p. 6.

[2] K. Bhattacharyya, S.M. Goodnick, J.F. Wager, J. Appl. Phys. 73, 3390 (1993).

[3] C. Jacoboni, R. Lugli, The Monte Carlo Method for Semiconductor Device Simulation, Springer-Verlag, New York 1989, p 38.

[4] K. Brennan, J. Appl. Phys. 64, 4024 (1988).

[5] B.K. Ridley, J. Phys. C, Solid State Phys. 16, 3373 (1983).

[6] E. Bringuier, J. Appl. Phys. 70, 4505 (1991).

[7] K. Brennan, J. Appl. Phys. 75, 678 (1994).

[8] R. Mach, J. Cryst. Growth 101, 967 (1990).

[9] H. Zhao, Y. Wang, Z. Xu, X. Xu, J. Phys, Condens. Matter 11, 2145 (1999).

[10] H. Zhao,. Y. Wang, Z. Xu, X. Xu, Science in China (E) 42, 282 (1999).

[11] W.E. Howard, O. Sahni, P.M. Alt, J. Appl. Phys. 53, 639 (1982).

[12] E. Bringuier, J. Appl. Phys. 70, 4505 (1991).

[13] E. Bringuier, Phys. Rev. B 49, 7974 (1994). 